\title{
Lung Ultrasound Prior to Spontaneous Breathing Trial Is Not Helpful in the Decision to Wean
}

\author{
Ana Carolina P Antonio MD PhD, Marli M Knorst MD PhD, and Cassiano Teixeira MD PhD
}

\begin{abstract}
BACKGROUND: Lung ultrasound is increasingly becoming a diagnostic tool in the critical care setting. B-pattern on a lung ultrasound is an artifact composed of multiple B-lines and correlates with interstitial edema. A randomized controlled trial concluded that bedside thoracic ultrasound could predict postextubation distress through changes in lung aeration during a weaning procedure; however, it could not screen patients before performance of a spontaneous breathing trial (SBT). METHODS: We conducted a 2-year, prospective, multicenter, observational study in 2 adult medical-surgical ICUs in southern Brazil. All enrolled subjects met eligibility criteria for ventilation liberation. Patients with tracheostomy were excluded. Lung ultrasound was performed immediately before SBT. B-predominance was defined as any profile with anterior bilateral B-pattern. The primary outcome was SBT failure, defined as the inability to tolerate a T-piece trial of 30-120 min, in which case subjects were not extubated. RESULTS: From 2011 to 2013, 250 subjects undergoing weaning procedures were evaluated. SBT failure occurred in $51(20.4 \%)$ subjects. Subjects with a successful SBT were extubated on the first attempt in $75.6 \%$ of cases. B-predominance was a very weak predictor for SBT outcome, showing $47 \%$ sensitivity, $64 \%$ specificity, $25 \%$ positive predictive value, and $82 \%$ negative predictive value. CONCLUSION: B-pattern detected by a simplified lung ultrasound protocol should not preclude hemodynamically stable, sufficiently oxygenated patients from performing an SBT. Key words: ventilator weaning; pulmonary edema; ultrasonography; clinical decision-making; spontaneous breathing trial. [Respir Care 2018;63(7):873-878. (C) 2018 Daedalus Enterprises]
\end{abstract}

\section{Introduction}

Liberation from mechanical ventilation remains one of the most challenging aspects of caring for critically ill patients. Clinical trials from the 1990s clearly showed that clinicians frequently did not appreciate that ventilator with-

\footnotetext{
Dr Antonio is affiliated with the Adult Intensive Care Unit of Hospital Moinhos de Vento, and Hospital de Clínicas de Porto Alegre, Porto Alegre, Brazil. Dr Knorst is affiliated with the Universidade Federal do Rio Grande do Sul. Dr Teixeira is affiliated with the Adult Intensive Care Unit of Hospital Moinhos de Vento, Porto Alegre, Brazil.
}

Dr Antonio presented a version of this paper at the 25th ESICM Annual Congress held October 13-17, 2012, in Lisbon, Portugal.

The authors have disclosed no conflicts of interest.

Correspondence: Ana Carolina P Antonio MD PhD, 910 Ramiro Barcelos, 3rd floor Porto Alegre, Brazil. E-mail: ana.carolina.antonio@gmail.com

DOI: $10.4187 /$ respcare.05817 drawal was possible in a timely fashion. ${ }^{1,2}$ Although lifesaving as long as needed, continuing mechanical ventilation is associated with increased morbidity and mortality, varying with age, the severity of preexisting and current disease processes, and ventilator plus interface (endotracheal or tracheostomy)-associated complications. ${ }^{3,4}$ On the other hand, failed or unplanned extubation is followed by marked clinical deterioration, suggesting a direct and specific effect of extubation failure and re-intubation on patient outcomes. ${ }^{5}$ The clinical challenge then is to balance aggressiveness with safety. ${ }^{6,7}$

Lung ultrasound has several advantages over conventional radiological means for assessing lung abnormalities in that it is reliable and accurate, highly reproducible, noninvasive, and easily repeatable at the bedside. ${ }^{8,9}$ B-lines are vertical hyperechoic sonographic artifacts that arise from the pleural line, extend to the bottom of the screen without fading, move synchronously with lung sliding, and erase A-lines. The presence or absence of B-lines is a useful tool for identifying pulmonary edema and alveolar 
interstitial syndrome in the acute setting, and there is evidence of real-time matching of B-line quantity with changes in extravascular lung water and total body water. ${ }^{10-15}$ Correlation between pulmonary artery occlusion pressure and B-lines in critically ill patients suggests it is a useful surrogate of left-sided filling pressures. ${ }^{12,16,17}$

Weaning-induced cardiac dysfunction is recognized as an important cause of weaning failure. ${ }^{18-20}$ Switching a patient from positive-pressure ventilation to spontaneous breathing reestablishes negative inspiratory intrathoracic pressure, thus increasing venous return (left ventricular preload), central blood volume, and left ventricular afterload. ${ }^{18,19}$ Increases in extravascular lung water induced by spontaneous breathing trials (SBTs) were detected in individuals who eventually failed weaning. ${ }^{21}$

In the last 20 years, from physiology to epidemiology, it seems that every imaginable factor with any potential to influence patients' ability to recover spontaneous breathing has been studied, and a large body of sometimes contradictory evidence has led to very different conclusions. ${ }^{22-24}$ One case report assessing both global and regional lung de-recruitment provided useful insights into the etiology of weaning failure. ${ }^{25}$ Soummer et $\mathrm{al}^{26} \mathrm{dem}$ onstrated that progressive lung de-recruitment during an SBT may accurately identify patients likely to fail extubation. However, as far as we know, no study has addressed the potential role of lung ultrasound findings in identifying individuals who are likely to fail to wean, so that either premature unsuccessful SBTs or undesirable delays could be avoided. Indeed, it is often unclear which factors should be considered in the decision-making process. Our previous study lacked sufficient power to detect any significant effects. ${ }^{27}$ Therefore, we sought to assess prospectively whether B-pattern of congestion seen on lung ultrasound prior to initiation of an SBT correlated with weaning outcomes in a heterogeneous group of mechanically ventilated subjects.

\section{Methods}

Between January 2011 and March 2013, nonconsecutive subjects $\geq 18$ y old who had undergone invasive mechanical ventilation for $24 \mathrm{~h}$ were enrolled from 2 medical-surgical ICUs in private hospitals in southern Brazil. Individuals with a tracheostomy were excluded. The research ethics board at each center approved the study and waived the requirement for informed consent. The study was registered as NCT02022839 at http://clinicaltrials.gov.

Subjects were assessed daily for eligibility to wean according to current guidelines, which included improvement of underlying condition that led to acute respiratory failure; alert and able to communicate; adequate gas exchange, as indicated by an arterial pressure of oxygen of at least $60 \mathrm{~mm} \mathrm{Hg}$ with an $\mathrm{F}_{\mathrm{IO}_{2}}<0.40$; no significant

\section{QUICK LOOK}

\section{Current knowledge}

Bedside lung ultrasound is a notable diagnostic tool in the ICU setting. B-lines are the sonographic sign for detecting pulmonary edema. Given the predictable negative physiological effects of pulmonary edema and large pleural effusions on lung mechanics, it is reasonable to consider whether checking for B-line predominance on lung ultrasound might be helpful to optimize the patient's condition before a spontaneous breathing trial (SBT) to increase the likelihood of successful extubation.

\section{What this paper contributes to our knowledge}

In 250 ready-to-wean individuals who underwent lung ultrasound immediately before an SBT, visualization of B-pattern bilaterally on the anterior chest performed poorly in predicting SBT failure. Lung ultrasound findings of B-predominance on a simplified 4-zone protocol should not interfere with the decision to initiate weaning procedures, as it does not identify individuals who will fail an SBT.

respiratory acidosis; rapid shallow breathing index $\leq 105$ breaths/min/L; and vasoactive drugs at low and stable doses (norepinephrine doses $<0.12 \mu \mathrm{g} / \mathrm{kg} / \mathrm{min}$ or equivalent dopamine doses). ${ }^{28,29}$ While local weaning protocols at both sites did not standardize PEEP levels as a prerequisite, it is a common practice to keep it $<8 \mathrm{~cm} \mathrm{H}_{2} \mathrm{O}$ before starting a T-piece trial.

A trained investigator using a 2- to 4-MHz convex probe performed lung ultrasounds as previously described.9,10,12,30 Subjects were scanned while in a semi-recumbent or supine position, immediately before starting the SBT. We divided the anterior thoracic surface into 4 areas, corresponding to the intercostal spaces between the third and fourth ribs and between the sixth and seventh ribs; the probe was inserted at the center of each, making 4 points of investigation per lung with dichotomous answer, requiring $<1 \mathrm{~min}$ (Fig. 1). Any profile with anterior bilateral B-pattern was denoted as B-predominance, which defines interstitial syndrome. ${ }^{9} \mathrm{We}$ did not describe patterns of aeration other than A-line, B-line, and C-line; the number of single or confluent B-lines was not reported.

The main outcome of interest was SBT failure, defined as an inability to tolerate a T-piece SBT of 30-120 min, in which case subjects were not extubated. The breathing trial was interrupted if subjects developed signs of respiratory discomfort (frequency $>35$ breaths $/ \mathrm{min}$, arterial oxyhemoglobin saturation $<90 \%$, use of accessory respiratory muscles or paradoxical thoracoabdominal ventila- 


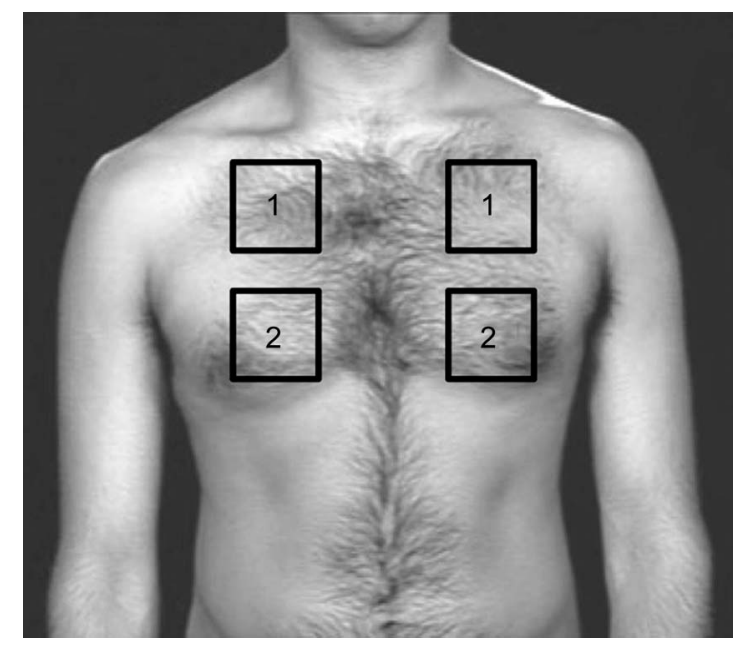

Fig. 1. The 4 parasternal views corresponding to the intercostal spaces between the third and fourth ribs and between the sixth and seventh ribs used to investigate B-pattern.

tion), tachycardia (heart rate $>140$ beats/min), hemodynamic instability (systolic blood pressure $<90 \mathrm{~mm} \mathrm{Hg}$ or $20 \%$ over baseline levels), or change in mental status (drowsiness, coma, anxiety). ${ }^{29}$ There were no secondary end points for this study.

Demographic data including age, gender, race, comorbidities, severity of illness at the time of ICU admission, reason for the initiation of mechanical ventilation, physiological weaning predictors, and fluid balance (total inputs minus total outputs) in the $48 \mathrm{~h}$ preceding the SBT were recorded. The presence of diastolic or systolic left ventricular dysfunction (the latter condition defined as ejection fraction $<45 \%$ ) was documented according to a formal echocardiogram report dated up to 6 months prior to admission. Diagnosis of COPD was based on history, physical examination, chest radiograph, and previous pulmonary function tests, if available.

\section{Statistics}

Results were expressed as the mean $\pm \mathrm{SD}$, median (interquartile range), or proportions, as appropriate. The normal distribution of the various parameters was investigated using the Kolmogorov-Smirnov test. We used the Student $t$ test or the Mann-Whitney $\mathrm{U}$ test to compare continuous variables, and the chi-square test or the Fisher exact test to compare proportions, as appropriate. Sensitivity, specificity, positive predictive value, negative predictive value, positive likelihood ratio, and negative likelihood ratio of B-predominance for the prediction of SBT failure were calculated. A $P$ value $<.05$ was considered statistically significant. Statistical analysis was performed with SPSS v.20.0 (SPSS, Chicago, Illinois).

\section{Results}

We obtained complete data in 250 subjects undergoing weaning procedures. Overall, failed SBT occurred in 51 $(20.4 \%)$ cases. Table 1 shows the baseline characteristics of the study cohort according to outcome. One hundred and eighty-nine individuals (75.6\%) were extubated on the first attempt - i.e., simple weaning procedures. Subjects who were successfully extubated were older (median 75 vs $66 \mathrm{y}, P=.03)$ and had been intubated for a shorter duration (median 4 vs $7 \mathrm{~d}, P<.001$ ). There was also a lower prevalence of COPD in the SBT success group (19.6\% vs $9.5 \%, P=.045)$.

B-predominance was observed in 95 cases, but only 24 subsequently failed SBT. Likewise, 27 subjects who did not succeed on SBT had showed no bilateral B-pattern prior to the T-piece SBT. B-predominance was a very weak predictor for SBT failure, as it provided sensitivity, specificity, positive predictive values, and negative predictive values of 0.47 (95\% CI 0.33-0.61), 0.64 (95\% CI $0.57-0.70), 0.25$ (95\% CI 0.17-0.35), and 0.82 (95\% CI $0.75-0.88)$, respectively.

Contrary to what was expected, individuals who exhibited B-predominance also had lower fluid balance in the $48 \mathrm{~h}$ prior to weaning procedure $(966[1,167.7-3,050] \mathrm{mL}$ vs $1,588[100-3,100] \mathrm{mL}, P=.043)$, possibly by chance. The large variability of values is shown in Figure 2.

\section{Discussion}

In a heterogeneous cohort of mechanically ventilated weaning candidates, the lung ultrasound finding of B-predominance on a simplified 4-zone protocol did not have the potential to alter decisions to start an SBT. Our data suggest that incorporating an ultrasonographic estimation of lung edema in the readiness criteria for withdrawal of mechanical ventilation potentially delays the decision.

The myriad changes in respiratory mechanics and cardiovascular status related to weaning failure do not become evident until clinical manifestations of distress, which promptly demand test interruption or re-intubation. ${ }^{31}$ Delaying SBT until B-lines wane (due to the belief that individuals could not pass a T-piece trial until they were dry again) has the potential to delay the overall weaning process. B-line artifacts may also be affected by other dynamic changes, such as the application of PEEP and the respiratory cycle, ${ }^{32}$ and we could not demonstrate any correlation between higher fluid balance and B-predominance. Fluid balance values did not predict SBT outcomes in a medical-surgical ICU population, and using pleural drainage to accelerate liberation from mechanical ventilation is also not supported by evidence. ${ }^{33,34}$

While we were in the process of gathering data for this study, Soummer et $\mathrm{al}^{26}$ reported that a quantitative assess- 
Table 1. Characteristics of the Study Cohort

\begin{tabular}{|c|c|c|c|}
\hline \multirow{2}{*}{ Subject Characteristics } & \multicolumn{2}{|c|}{ Spontaneous Breathing Trial } & \multirow{2}{*}{$P$} \\
\hline & Success $(n=199)$ & Failure $(n=51)$ & \\
\hline Age, y & $75(60-83)$ & $66(47-81)$ & .03 \\
\hline Female gender, $\%$ & 42.2 & 45.1 & .71 \\
\hline APACHE II, points & $20.8 \pm 6.9$ & $21.8 \pm 7.8$ & .25 \\
\hline SOFA score, points & $5(3-9)$ & $5(2-9)$ & .89 \\
\hline Body mass index, $\mathrm{kg} / \mathrm{m}^{2}$ & $25(23-30)$ & $25(23-29)$ & .82 \\
\hline Rapid shallow breathing index, $f / V_{T}$ & $58.3 \pm 24.3$ & $58.4 \pm 24$ & .89 \\
\hline Mechanical ventilation duration, $\mathrm{d}$ & $4(2-6)$ & $7(4-11)$ & $<.001$ \\
\hline \multicolumn{4}{|l|}{ Comorbidities, $n(\%)$} \\
\hline COPD & $19(9.5)$ & $10(19.6)$ & .03 \\
\hline Ejection fraction $<45 \%$ & $19(9.5)$ & $6(11.8)$ & .64 \\
\hline Left ventricular diastolic dysfunction & $73(65.8)$ & $15(51.7)$ & .16 \\
\hline Ischemic coronary disease & $36(18.1)$ & $5(9.8)$ & .15 \\
\hline Renal replacement therapy & $34(17.1)$ & $13(25.5)$ & .17 \\
\hline Presence of ascitis & $7(3.5)$ & $3(5.9)$ & .44 \\
\hline \multicolumn{4}{|l|}{ Reason for mechanical ventilation, $n(\%)$} \\
\hline Respiratory sepsis & $31(15.6)$ & $9(17.6)$ & .72 \\
\hline Non-respiratory sepsis & $52(26.1)$ & $12(23.5)$ & .70 \\
\hline Congestive heart failure & $20(10.1)$ & $2(3.9)$ & .17 \\
\hline Coma & 39 (19.6) & $6(11.8)$ & .19 \\
\hline Postoperative acute respiratory failure & $15(7.5)$ & $4(7.8)$ & .94 \\
\hline COPD/asthma & $2(1)$ & $2(3.9)$ & .14 \\
\hline Pulmonary embolism & $6(3)$ & $1(2)$ & .68 \\
\hline ARDS & $10(5)$ & $5(9.8)$ & .20 \\
\hline Simple weaning, $n(\%) *$ & $151(75.9)$ & $38(74.5)$ & .61 \\
\hline Chest radiograph pleural effusion, $n(\%)$ & $60(30.2)$ & $17(33.3)$ & .66 \\
\hline Chest radiograph pulmonary edema, $n(\%)$ & $56(26.6)$ & $18(35.3)$ & .22 \\
\hline Vasopressor infusion during T-piece trial, $n(\%)$ & $39(19.6)$ & $5(9.8)$ & .10 \\
\hline Vasodilator infusion during T-piece trial, $n(\%)$ & $13(6.5)$ & $3(5.9)$ & .87 \\
\hline $48 \mathrm{~h}$-fluid balance prior to T-piece trial, $\mathrm{mL}$ & $1,324.39 \pm 2,915.95$ & $1,201.65 \pm 2,801.68$ & .52 \\
\hline \multicolumn{4}{|c|}{$\begin{array}{l}\text { Data are presented as median (interquartile range), mean } \pm \mathrm{SD} \text {, or } n(\%) . N=250 \text { spontaneous breathing trials. } \\
\text { * Simple weaning was defined as proceeding from initiation of weaning to successful extubation on the first attempt without difficulty (from Reference 28). } \\
\text { APACHE II }=\text { Acute Physiology and Chronic Health Evaluation II } \\
\text { SOFA = Sequential Organ Failure Assessment }\end{array}$} \\
\hline
\end{tabular}

ment of 6 chest regions with lung ultrasound accurately predicted extubation failure by identifying global and regional lung derecruitment at the end of SBT. They speculated that subjects who eventually required re-intubation had either greater tendency to pulmonary collapse or lack of muscle endurance. However, the authors were unable to anticipate 14 individuals who failed on a 60-min T-piece trial and thus were not extubated; lung ultrasound scores before SBTs were not different between groups. ${ }^{26}$

In a preliminary report, ${ }^{27}$ we postulated that a simplified approach using 4 anterior chest zones would be adequate for the specific purpose of our study because the B-pattern or consolidation found at lower and posterior lung regions was likely to indicate gravitational changes. Such a 4-zone protocol has been successfully adopted for other purposes elsewhere. ${ }^{10,13,16,35}$ Indeed, reducing scanning to just 4 anterior chest zones facilitated initial assessment of this sub- set of subjects through a simple, inexpensive, safe, rapid, and easy-to-perform screening test. ${ }^{9,10,30} \mathrm{Within}$ up to $1 \mathrm{~min}$ of lung ultrasound examination, researchers were able to reliably identify B-pattern. The counting approach may be imprecise when considering single scanning sites, and although it has utility as a research tool, it may be overly complicated for the front-line intensivist to use in a busy ICU. No conclusions could be drawn regarding more precise quantification of interstitial syndrome and the relationship to SBT outcomes using different protocols, such as an 8-, 12-, or even a 28-zone approach.

We chose SBT failure as the principal outcome because we aimed to predict the earliest time that a patient might resume spontaneous breathing. Moreover, the exact reason for extubation failure often escapes identification. Reintubation is usually performed because of an apparently new episode of respiratory distress, which may be related 


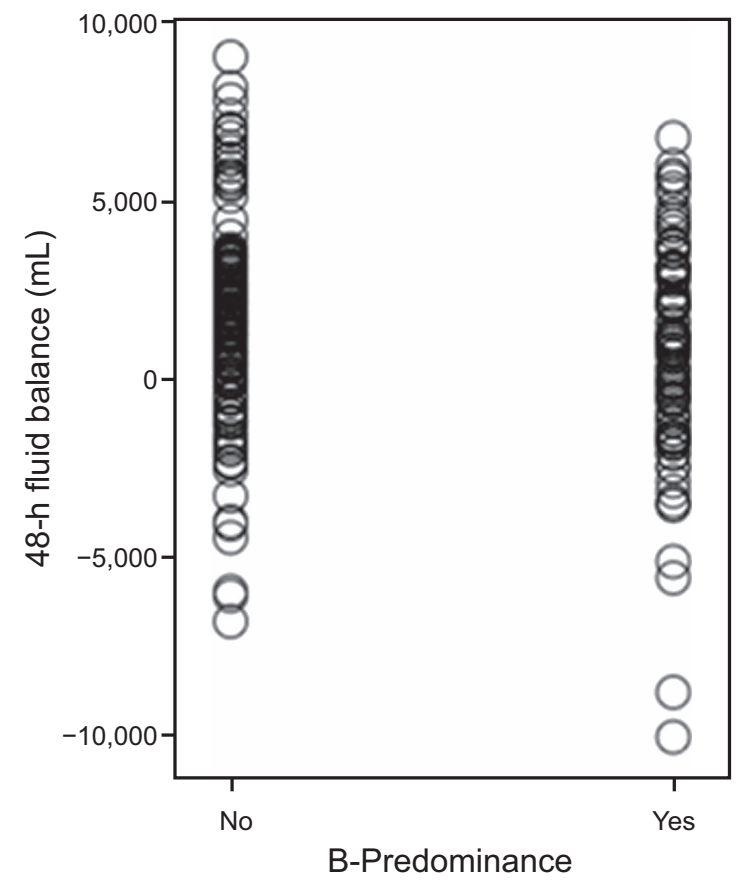

Fig. 2. Range of values of fluid balance in the preceding $48 \mathrm{~h}$ of spontaneous breathing trials according to finding of B-predominance on lung ultrasound. Median (range) fluid balance was lower in the B-predominance group, although there was a wide range of values: $966(1,167.7-3,050) \mathrm{mL}$ vs $1,588(100-3,100) \mathrm{mL}, P=.043$.

to primary respiratory failure, congestive heart failure, aspiration, ineffective cough with airway secretion build-up, upper airway obstruction, onset of new sepsis, surgical complications, acute coronary syndrome, or neurological impairment. This multiplicity of causative factors explain persistent uncertainties about the pathophysiology of extubation failure. ${ }^{31}$

Limitations of our study were its observational design, its intrinsic methodological flaws, and its small sample size. Lung ultrasound examinations were performed only during working hours. The choice of a convenience sample also limits the interpretation and generalization of the findings. The prevalence of simple weaning $(75.6 \%)$ indicates that our prospective opportunity sample had same expected pre-test probability of SBT failure as any ordinary, medical-surgical ICU population..$^{28,29,31}$ Simple weaning was defined as proceeding from initiation of weaning to successful extubation on the first attempt without difficulty. ${ }^{28}$ No echocardiographic data were obtained; however, because increases in respiratory load and cardiac load are strongly interrelated, it is difficult to estimate the real incidence of cardiac-related weaning failure. ${ }^{18,19} \mathrm{Nei}-$ ther transthoracic echocardiogram changes nor brain natriuretic peptide levels provided clinically useful cutoffs for predicting postextubation distress in a previous study. ${ }^{26}$ Only 24 (9.6\%) of subjects included in our study had a history of cardiac failure, thus no inference could be made.
Like all ultrasound techniques, bedside lung-ultrasound quality can be operator-dependent; however, a high intraand inter-observer reproducibility has been reported. ${ }^{36}$

One may wonder whether it is really pertinent to predict results of a simple and safe test such as an SBT rather than to simply perform it. There is no definitive evidence that a carefully monitored but unsuccessful SBT is detrimental to weaning outcome. To the contrary, in a study of 1,067 subjects undergoing daily screens, only a one major complication $(0.1 \%)$ could be possibly attributed to a failed SBT. ${ }^{37}$ Therefore, in our opinion, there is no real need to perform lung ultrasound simply to avoid proceeding to an SBT in individuals already meeting eligibility criteria for weaning.

\section{Conclusion}

The B-pattern detected by a simplified lung ultrasound protocol should not preclude hemodynamically stable and sufficiently oxygenated patients from performing an SBT. Likelihood ratios are not high enough to serve as standalone criteria for clinical decision making. Lung ultrasounds may be helpful in defining a mechanism for weaning failure and in guiding intervention that may increase the success of the next attempt, particularly when integrated with echocardiography. A multicenter, randomized, interventional study is required to assess the impact of an ultrasound algorithm on weaning rate, length of stay, and ICU mortality.

\section{REFERENCES}

1. Brochard L, Rauss A, Benito S, Conti G, Mancebo J, Rekik N, et al. Comparison of three methods of gradual withdrawal from ventilatory support during weaning from mechanical ventilation. Am J Respir Crit Care Med 1994;150(4):896-903.

2. Esteban A, Frutos F, Tobin MJ, Alia I, Solsona JF, Valverdu I, et al. A comparison of four methods of weaning patients from mechanical ventilation. Spanish Lung Failure Collaborative Group. N Engl J Med 1995;332(6):345-350.

3. Ely EW, Baker AM, Dunagan DP, Burke HL, Smith AC, Kelly PT, et al. Effect on the duration of mechanical ventilation of identifying patients capable of breathing spontaneously. N Engl J Med 1996; 335(25):1864-1869.

4. Cook DJ, Walter SD, Cook RJ, Griffith LE, Guyatt GH, Leasa D, et al. Incidence of and risk factors for ventilator-associated pneumonia in critically ill patients. Ann Intern Med 1998;129(6):433-440.

5. Thille AW, Harrois A, Schortgen F, Brun-Buisson C, Brochard L. Outcomes of extubation failure in medical intensive care unit patients. Crit Care Med 2011;39(12):2612-2618.

6. Krinsley JS, Reddy PK, Iqbal A. What is the optimal rate of failed extubation? Crit Care 2012;16(1):111.

7. MacIntyre NR. The ventilator discontinuation process: an expanding evidence base. Respir Care 2013;58(6):1074-1086.

8. Lichtenstein DA. Lung ultrasound in the critically ill. Ann Intensive Care 2014;4(1):1.

9. Volpicelli G, Elbarbary M, Blaivas M, Lichtenstein DA, Mathis G, Kirkpatrick AW, et al. International evidence-based recommenda- 


\section{Lung Ultrasound Prior to SBT and Decision to Wean}

tions for point-of-care lung ultrasound. Intensive Care Med 2012; 38(4):577-591.

10. Lichtenstein DA, Meziere GA. Relevance of lung ultrasound in the diagnosis of acute respiratory failure: the BLUE protocol. Chest 2008;134(1):117-125.

11. Volpicelli G, Mussa A, Garofalo G, Cardinale L, Casoli G, Perotto $\mathrm{F}$, et al. Bedside lung ultrasound in the assessment of alveolarinterstitial syndrome. Am J Emerg Med 2006;24(6):689-696.

12. Agricola E, Bove T, Oppizzi M, Marino G, Zangrillo A, Margonato A, et al. "Ultrasound comet-tail images": a marker of pulmonary edema: a comparative study with wedge pressure and extravascular lung water. Chest 2005;127(5):1690-1695.

13. Enghard P, Rademacher S, Nee J, Hasper D, Engert U, Jorres A, et al. Simplified lung ultrasound protocol shows excellent prediction of extravascular lung water in ventilated intensive care patients. Crit Care 2015;19:36.

14. Noble VE, Murray AF, Capp R, Sylvia-Reardon MH, Steele DJ, Liteplo A. Ultrasound assessment for extravascular lung water in patients undergoing hemodialysis. Time course for resolution. Chest 2009;135(6):1433-1439.

15. Volpicelli G, Skurzak S, Boero E, Carpinteri G, Tengattini M, Stefanone $\mathrm{V}$, et al. Lung ultrasound predicts well extravascular lung water but is of limited usefulness in the prediction of wedge pressure. Anesthesiology 2014;121(2):320-327.

16. Lichtenstein DA, Meziere GA, Lagoueyte JF, Biderman P, Goldstein I, Gepner A. A-lines and B-lines: lung ultrasound as a bedside tool for predicting pulmonary artery occlusion pressure in the critically ill. Chest 2009;136(4):1014-1020.

17. Lichtenstein D. Fluid administration limited by lung sonography: the place of lung ultrasound in assessment of acute circulatory failure (the FALLS-protocol). Expert Rev Respir Med 2012;6(2):155-162.

18. Teboul JL. Weaning-induced cardiac dysfunction: where are we today? Intensive Care Med 2014;40(8):1069-1079.

19. Teboul JL, Monnet X, Richard C. Weaning failure of cardiac origin: recent advances. Crit Care 2010;14(2):211.

20. Cabello B, Thille AW, Roche-Campo F, Brochard L, Gomez FJ, Mancebo J. Physiological comparison of three spontaneous breathing trials in difficult-to-wean patients. Intensive Care Med 2010; 36(7):1171-1179.

21. Dres M, Teboul JL, Anguel N, Guerin L, Richard C, Monnet X. Extravascular lung water, B-type natriuretic peptide, and blood volume contraction enable diagnosis of weaning-induced pulmonary edema. Crit Care Med 2014;42(8):1882-1889.

22. Teixeira C, Teixeira PJ, de Leon PP, Oliveira ES. Work of breathing during successful spontaneous breathing trial. J Crit Care 2009;24(4): 508-514.

23. Meade M, Guyatt G, Cook D, Griffith L, Sinuff T, Kergl C, et al. Predicting success in weaning from mechanical ventilation. Chest 2001;120(6 Suppl):400S-424S.

24. Tanios MA, Nevins ML, Hendra KP, Cardinal P, Allan JE, Naumova $\mathrm{EN}$, et al. A randomized, controlled trial of the role of weaning predictors in clinical decision making. Crit Care Med 2006;34(10): 2530-2535.

25. Mongodi S, Via G, Bouhemad B, Storti E, Mojoli F, Braschi A Usefulness of combined bedside lung ultrasound and echocardiography to assess weaning failure from mechanical ventilation: a suggestive case. Crit Care Med 2013;41(8):e182-e185.

26. Soummer A, Perbet S, Brisson H, Arbelot C, Constantin JM, Lu Q, et al. Ultrasound assessment of lung aeration loss during a successful weaning trial predicts postextubation distress. Crit Care Med 2012; 40(7):2064-2072.

27. Antonio ACP, Teixeira C, Castro PS, Savi A, Maccari JG, Oliveira $\mathrm{RP}$, et al. Behavior of lung ultrasound findings during spontaneous breathing trial. Rev Bras Ter Intensiva 2017;29(3):279-286.

28. MacIntyre NR, Cook DJ, Ely EW Jr., Epstein SK, Fink JB, Heffner $\mathrm{JE}$, et al. Evidence-based guidelines for weaning and discontinuing ventilatory support: a collective task force facilitated by the American College of Chest Physicians; the American Association for Respir Care; and the American College of Crit Care Med Chest. 2001; 120(6 Suppl):375S-395S.

29. Boles JM, Bion J, Connors A, Herridge M, Marsh B, Melot C, et al. Weaning from mechanical ventilation. Eur Respir J 2007;29(5): 10331056.

30. Gargani L, Volpicelli G. How I do it: lung ultrasound. Cardiovasc Ultrasound 2014;12:25.

31. Tobin M, A J. Weaning from mechanical ventilation. In: Tobin M, ed. Principles and Practice of Mechanical Ventilation, 3rd ed. New York: McGraw-Hill; 2012:1185-1220.

32. Bouhemad B, Brisson H, Le-Guen M, Arbelot C, Lu Q, Rouby JJ. Bedside ultrasound assessment of positive end-expiratory pressureinduced lung recruitment. Am J Respir Crit Care Med 2011;183(3): 341-347.

33. Antonio AC, Teixeira C, Castro PS, Savi A, Oliveira RP, Gazzana MB, et al. 48-Hour fluid balance does not predict a successful spontaneous breathing trial. Respir Care 2015;60(8):1091-1096.

34. Goligher EC, Leis JA, Fowler RA, Pinto R, Adhikari NK, Ferguson ND. Utility and safety of draining pleural effusions in mechanically ventilated patients: a systematic review and meta-analysis. Crit Care 2011;15(1):R46.

35. Santos TM, Franci D, Coutinho CM, Ribeiro DL, Schweller M, Matos-Souza JR, et al. A simplified ultrasound-based edema score to assess lung injury and clinical severity in septic patients. Am J Emerg Med 2013;31(12):1656-1660.

36. Bouhemad B, Liu ZH, Arbelot C, Zhang M, Ferarri F, Le-Guen M, et al. Ultrasound assessment of antibiotic-induced pulmonary reaeration in ventilator-associated pneumonia. Crit Care Med 2010;38(1): 84-92.

37. Ely EW, Bennett PA, Bowton DL, Murphy SM, Florance AM, Haponik EF. Large scale implementation of a respiratory therapistdriven protocol for ventilator weaning. Am J Respir Crit Care Med 1999;159(2):439-446. 